\title{
QUASI-PERIODIC OSCILLATIONS IN THE X-RAY LIGHT CURVES FROM RELATIVISTIC TORI
}

\author{
JEREMY D. SchnitTMAN ${ }^{1,2}$ AND Luciano Rezzolla ${ }^{3,4,5}$ \\ Received 2005 June 28; accepted 2005 December 12; published 2006 January 18
}

\begin{abstract}
We use a relativistic ray-tracing code coupled to a relativistic hydrodynamics code to analyze the X-ray emission from a pressure-supported oscillating torus around a black hole. Solving the relativistic radiative transfer equation along each photon path, we calculate the X-ray light curves, power spectra, and broadened emission lines, as seen by a distant observer from various inclinations. We show that a strong correlation exists between the intrinsic frequencies of the torus normal modes and the extrinsic frequencies seen in the observed light-curve power spectrum. This correlation demonstrates the feasibility of the oscillating-torus model to explain the multiple peaks seen in black hole high-frequency quasi-periodic oscillations. Observations of these features can provide important information about the torus as well as the black hole.
\end{abstract}

Subject headings: accretion, accretion disks — black hole physics - X-rays: binaries

Online material: color figure

\section{INTRODUCTION}

Recent observations with the Rossi X-Ray Timing Explorer $(R X T E)$ have revealed the existence of high-frequency quasiperiodic oscillations (QPOs) in multiple accreting black hole binary systems (Strohmayer 2001). In an increasing number of these systems, the QPOs appear with integer commensurabilities, generally a 2:3 frequency ratio (Miller et al. 2001; Remillard et al. 2002; Homan et al. 2005). Since these modulations are expected to originate very close to the black hole, they could be used to test gravity in strong-field regimes or extract information on the black hole properties.

Over the years since their discovery, a large number of theoretical models have been developed to explain these observations. Some of the more popular models explain the QPOs through fluid oscillations in thin disks (Okazaki et al. 1987; Nowak et al. 1997), geodesic resonances (Stella \& Vietri 1999; Abramowicz \& Kluźniak 2001), and trapped fluid oscillations in pressure-supported tori (Rezzolla et al. 2003a; Lee et al. 2004).

To evaluate the relative strengths and weaknesses of any of these models, it is essential to compare directly the predictions of the theoretical models with the observations. To this end, we have coupled the ray-tracing methods described in Schnittman \& Bertschinger (2004) with the relativistic hydrodynamics code described in Zanotti et al. (2003). Using the hydrodynamic fluid properties of the simulated axisymmetric torus, we solve the relativistic radiative transfer equation along each photon path. These calculations represent the first light curves and spectra from self-consistent general relativistic hydrodynamic simulations of an accreting black hole. For three different emission models we have calculated X-ray spectra, light curves, and QPO power spectra, all as measured by a distant observer. In this proof-of-principle calculation, we demonstrate that a

\footnotetext{
${ }^{1}$ Department of Physics, University of Maryland, 82 Regents Drive, College Park, MD 20742; schnittm@umd.edu.

${ }^{2}$ Department of Physics, Massachusetts Institute of Technology, 77 Massachusetts Avenue, Cambridge, MA 02139.

${ }^{3}$ Max-Planck-Institut für Gravitationsphysik (Albert-Einstein-Institut), Am Mühlenberg 1, D-14476 Golm, Germany; rezzolla@aei.mpg.de.

${ }^{4}$ Scuola Internazionale Superiore di Studi Avanzati and Istituto Nazionale de Fizica Nucleare, via Beirut 2, I-34014 Trieste, Italy.

${ }^{5}$ Department of Physics and Astronomy, Louisiana State University, 202 Nicholson Hall, Baton Rouge, LA 70803.
}

strong correlation exists between the intrinsic normal-mode oscillations of a pressure-supported torus and the extrinsic observables of the light curves and power spectra. Our analysis is fundamentally different from that of Bursa et al. (2004), where the torus model was strictly analytical and not the result of self-consistent hydrodynamic simulations.

This Letter is organized as follows: In $\S 2$, we summarize the basic dynamical features of the torus simulations and describe how we apply the classical radiative transfer equation to a general relativistic fluid. In $\S 3$, we calculate X-ray light curves and the associated QPO power spectra for the different emission models over a range of inclinations, describing the major relativistic effects involved. We conclude in $\S 4$ with a discussion of the major results and a look toward future work.

\section{TORUS DYNAMICS AND RADIATIVE TRANSFER}

To briefly summarize the basic properties of the oscillatingtorus model, we recall that we are considering a non-selfgravitating perfect-fluid torus orbiting a Schwarzschild black hole (Font \& Daigne 2002; Zanotti et al. 2003). The fluid is assumed to be in circular, nongeodesic motion, and the conditions of hydrostatic equilibrium and of azimuthal symmetry allow the relativistic hydrodynamics equations to be reduced to Bernoulli-type equations. These have particularly simple solutions when the fluid is assumed to have a constant specific angular momentum and a polytropic equation of state is adopted. In this case, the equations of hydrostatic equilibrium can be integrated analytically to yield the rest-mass density distribution inside the torus, with the isobaric surfaces coinciding with the equipotential ones. Hereafter we will consider tori with constant specific angular momentum and bear in mind that more complex distributions introduce only small quantitative differences (for details, see Montero et al. 2004; Zanotti et al. 2005).

Once a stationary equilibrium configuration is constructed, it is perturbed with the introduction of a small radial velocity proportional to the radial velocity $v_{\text {Michel }}$ for a spherically symmetric accretion flow onto a Schwarzschild black hole (Michel 1972). We set the initial fluid 3-velocity as $v_{r}=\eta v_{\text {Michel }}$ and then use the dimensionless coefficient $\eta$ to tune the strength of the perturbation, obtaining an essentially linear response for $\eta \lesssim 0.06$ (Zanotti et al. 2003). With these initial conditions, 
the equations of relativistic hydrodynamics in a Schwarzschild black hole spacetime are solved using the axisymmetric, general relativistic code described in Zanotti et al. (2003, 2005).

The introduction of the perturbations triggers harmonic oscillations of the torus, with centrifugal forces and pressure gradients acting to restore equilibrium. A careful investigation of these oscillations has also revealed that there are multiple peaks in the power spectrum with frequencies in a sequence of integers: $2: 3: 4: \cdots$ (Zanotti et al. 2003, 2005). Subsequent perturbative analyses have also shown that these oscillations are indeed $p$-modes, behaving as trapped waves within the cavity produced by the torus and hence having eigenfrequencies in a sequence of small integers (Rezzolla et al. 2003b; Montero et al. 2004). The striking analogy between the harmonic relation among the $p$-mode eigenfrequencies and the QPOs observed in black hole systems has led to the suggestion that QPOs could result from basic fluid oscillations of a small accretion torus close to the black hole (Rezzolla et al. 2003a).

While attractive for its simplicity and for being based on global modes of oscillation that are expected to be present in realistic accretion disks, the relativistic torus model has so far only suggested a property of an orbiting, pressure-supported gas itself. It is not intuitively obvious how "intrinsic" modulations in the fluid hydrodynamics will produce similar "extrinsic" modulations in the observed light curve. Furthermore, it is not clear a priori what might be the relationship between the phases and amplitudes of the intrinsic and extrinsic oscillations, and how these might compare with the X-ray data.

To explore this relationship quantitatively, we have calculated the trajectories of photons from a distant observer to the emission region around a black hole following the methods described in Schnittman \& Bertschinger (2004). In this approach, the photon positions and momenta are tabulated along each ray's path in order to recreate a simulated, time-varying image of the accreting gas. Given the photon's 4-position and 4-momentum along the entire path, the observed spectrum is calculated for that ray by integrating the radiative transfer equation with a model for the gas emissivity and absorption.

More precisely, we start from the classical radiative transfer equation

$$
\frac{d I_{v}}{d s}=j_{\nu}-\alpha_{\nu} I_{\nu}
$$

(Rybicki \& Lightman 1979), where $d s$ is the differential path length and $I_{\nu}, j_{\nu}$, and $\alpha_{\nu}$ are respectively the spectral intensity, emissivity, and absorption coefficient of the fluid at a frequency $\nu$. The absorption coefficient is related to the opacity $\kappa_{\nu}$ through the density $\rho: \alpha_{\nu}=\rho \kappa_{\nu}$. These variables are generally functions of the gas temperature and density, defined in the local fluid frame. We use three different emission models in this Letter: (1) optically thin line emission at frequency $\nu_{\mathrm{em}}$ with $\alpha_{\nu}=0$ and $j_{\nu} \propto \rho \delta\left(\nu-\nu_{\text {em }}\right)$; (2) optically thick line emission and absorption, with both $\alpha_{\nu}$ and $j_{\nu}$ proportional to $\rho \delta\left(\nu-\nu_{\mathrm{em}}\right)$; and (3) optically thick thermal emission and absorption, using a Kramers opacity law with

$$
\kappa_{\nu} \propto \rho T^{-7 / 2}\left(\frac{1-e^{-x}}{x^{3}}\right), \quad j_{\nu} \propto \rho^{2} T^{-1 / 2} e^{-x},
$$

where $x \equiv h \nu / k T$. For the thermal emission model, the temperature is derived from the simulation variables assuming radiation-
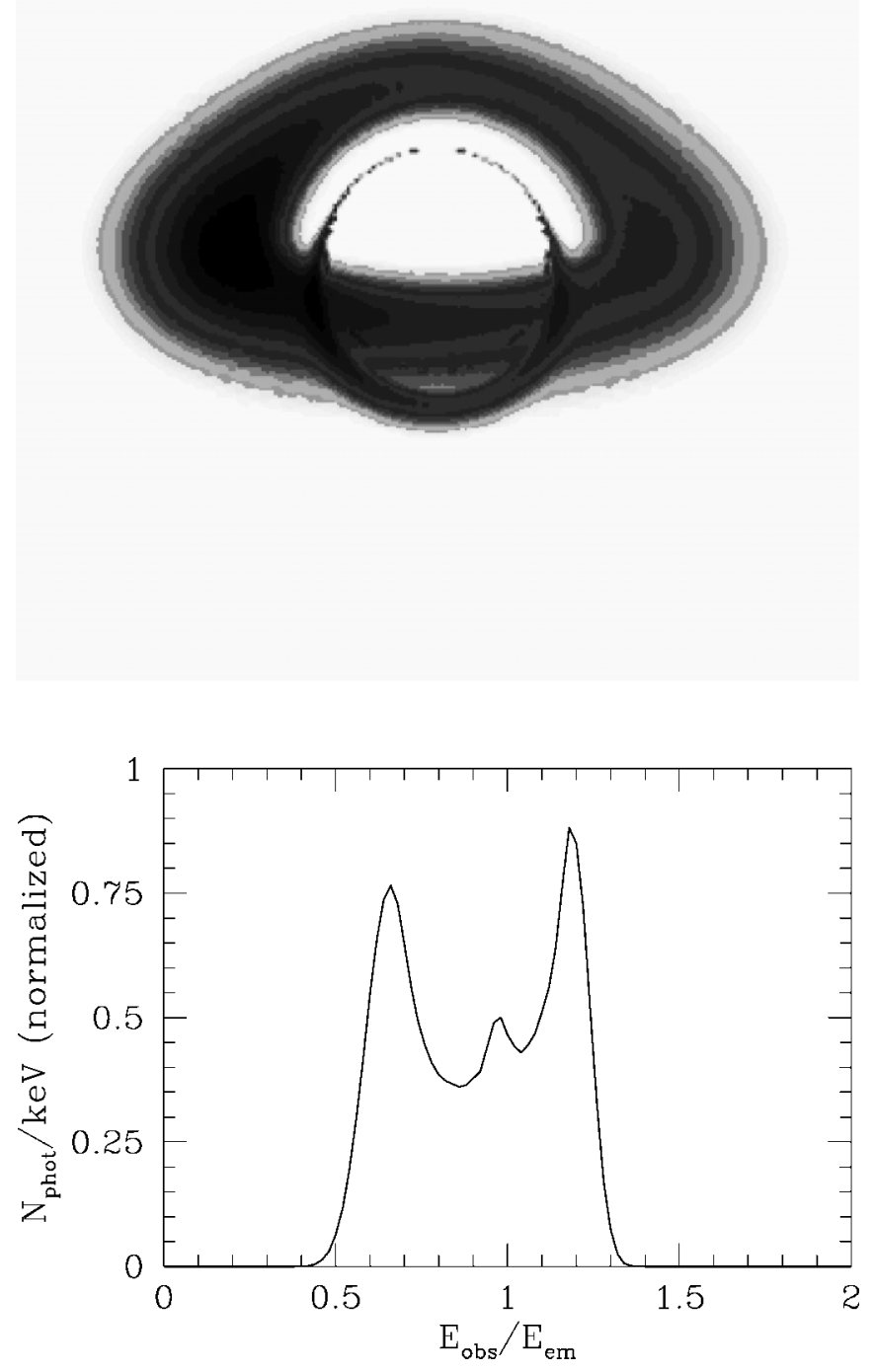

FIG. 1.-Top: Ray-traced image of the oscillating torus, for an inclination $i=70^{\circ}$, with a logarithmic scale for the X-ray intensity. Bottom: Broadened line spectrum for the optically thin emission model. The triple-peaked spectrum is caused by relativistic beaming toward and away from the observer, along with a central peak due to gravitational lensing of the far side of the accretion torus. [See the electronic edition of the Journal for a color version of this figure.]

dominated pressure and a black hole mass $M=10 M_{\odot}$, giving typical gas temperatures of $T \sim 10^{7} \mathrm{~K}$.

We incorporate relativistic effects by defining a local orthonormal tetrad at each point along the integration path (for the Schwarzschild geometry, this is simply the coordinatestationary observer frame). Once transformed to this locally flat tetrad, only special relativistic effects must be included. Following Rybicki \& Lightman (1979), equation (1) becomes

$$
\frac{d I_{\nu}}{d s}=\left(\frac{\nu}{\nu^{\prime}}\right)^{2} j_{\nu}^{\prime}-\left(\frac{\nu^{\prime}}{\nu}\right) \alpha_{\nu}^{\prime} I_{\nu},
$$

where primed and unprimed variables are measured in the rest frame of the gas and in the stationary tetrad, respectively. In 
addition, as the photon bundle propagates through the global curvature around the black hole, the spectral intensity at a given frequency evolves as the photons are gravitationally redshifted, maintaining the Lorentz invariance of $I_{\nu} / \nu^{3}$.

Figure 1 shows a simulated image of the torus, observed with inclination $i=70^{\circ}$, using the optically thin line emission model, which clearly highlights the relativistic effects involved. We also plot the corresponding broadened emission line, showing the two characteristic peaks caused by the Doppler shift of gas moving toward and away from the observer. For this geometry, a third, intermediate peak is also seen, caused by the gravitational magnification of a small region of the torus on the far side of the black hole (Beckwith \& Done 2004; Schnittman et al. 2006).

\section{LIGHT CURVES AND POWER SPECTRA}

In Figure 2, we plot the light curves for a torus orbiting around a Schwarzschild black hole of mass $M=10 M_{\odot}$ at inclination angles of $10^{\circ}$ and $70^{\circ}$, for the three emission models described above. The amplitude of the initial perturbation was $\eta=0.02$, but similar behavior is seen for perturbations throughout the linear regime, that is, $\delta I / I \propto \eta$ for $\eta \lesssim 0.06$, where $I \equiv \int_{0}^{\infty} I_{\nu} d \nu$. Although it is clear that the light curve has a quasi-periodic behavior and we find that this is strictly related to the oscillating behavior of the torus at the same frequencies, the quantitative features are the combination of several different physical effects. For the line emission models, the minimum of the intensity is reached in the "compression" phase of the oscillation, when the torus is smaller in size and closer to the black hole. In this case the gravitational redshift reduces the observed intensity, as the light has to escape from a deeper potential. The variation is even greater for the optically thick case, where the total flux is proportional to the projected area of the torus, which also decreases with radius. However, for the thermal model the emission is actually at maximum for a smaller radius, as the temperature of the compressed torus increases adiabatically, more than compensating for the gravitational redshift.

For all three models, the intensity is also varied by the special relativistic beaming of photons emitted toward and away from the observer. Smaller contributions to the intensity modulation also come from the transverse ("second-order") Doppler shift and from the gravitational lensing of the far side of the torus. The size of the third, intermediate peak in Figure $1 b$ is a testament to the relatively small contribution from gravitational lensing. Because of the conservation of angular momentum during the oscillations, the fluid velocities increase as the torus approaches the black hole, thus enhancing the relativistic beaming of photons toward the observer. For large inclination angles (i.e., when the torus is almost "edge-on"), this beaming serves to compensate for the intensity decrease due to the gravitational redshift. As a result, the two major relativistic effects counter each other and the intensity modulation is smaller for the line emission models (compare dashed and solid lines in Figs. $2 a$ and $2 b$ ). Again, the opposite behavior is seen in the thermal model: the relativistic beaming enhances the emission for smaller radii, giving larger amplitude fluctuations for high inclinations (see Fig. 2c).

It is important to note that for the optically thin line emission model, the dependence of the light-curve modulation on the inclination angle is the qualitative opposite of the hot-spot model described in Schnittman \& Bertschinger (2004), which

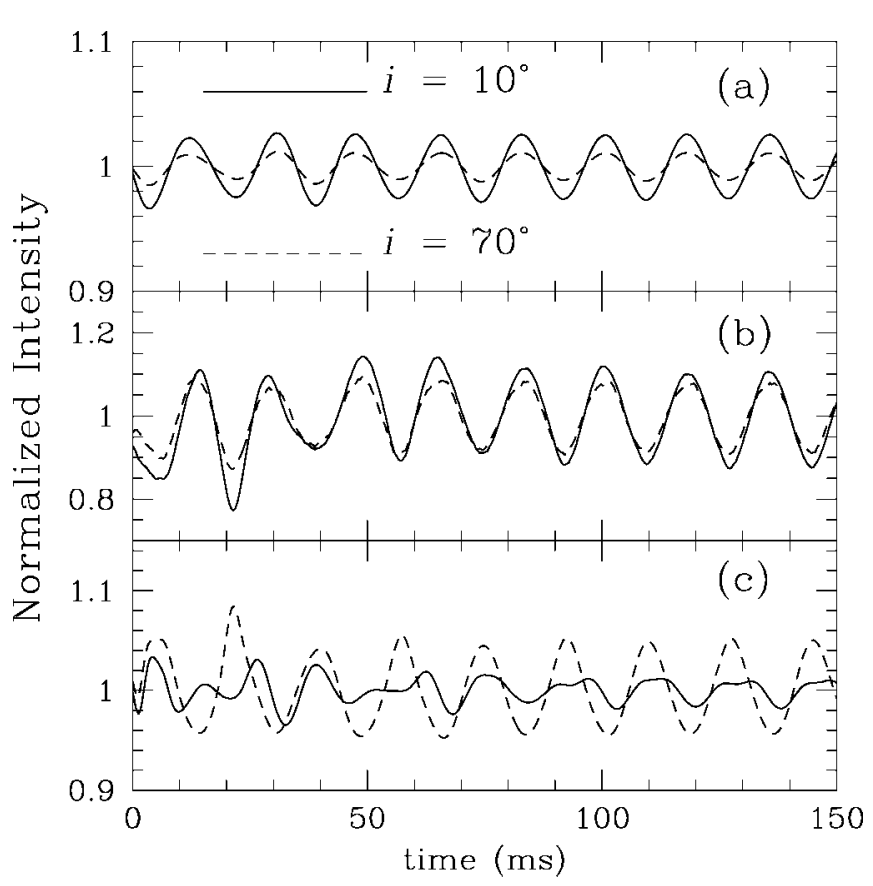

FIG. 2.-Normalized X-ray light curves from oscillating tori with inclinations $i=10^{\circ}$ (solid curves) and $i=70^{\circ}$ (dashed curves), for three different emission models: $(a)$ optically thin line emission, $(b)$ optically thick line emission and absorption, and $(c)$ optically thick thermal emission and absorption. The black hole has mass $M=10 M_{\odot}$, and the torus perturbation amplitude is $\eta=0.02$.

uses essentially the same emission model. Furthermore, the significant difference between all the light curves plotted in Figure 2 emphasizes the need for detailed radiative transfer calculations: the same intrinsic torus modulations give extrinsic modulations with a wide variety of amplitudes and phases (yet all at the same frequencies). These differences could serve to distinguish between the different models as more observations become available.

In Figure 3, we show the power spectra from the light curves plotted in Figure $2 a$, in units of (rms/mean) $)^{2} \mathrm{~Hz}^{-1}$. The power is clearly dominated by a peak at $55 \mathrm{~Hz}$, which coincides with the lowest $p$-mode of the torus (indicated as the fundamental, $f)$. Furthermore, the power spectra also show smaller peaks at the overtones $o_{1} \simeq(3 / 2) f, o_{2} \simeq 2 f$, and $o_{3} \simeq(5 / 2) f$, thus demonstrating that the light curve possesses the same harmonic behavior as the underlining hydrodynamics. Power spectra for the other emission models appear similar, with slightly more harmonic power present in the thermal model, as suggested by the nonsinusoidal nature of the light curves in Figure $2 c$. In order to ultimately match the observed QPO amplitudes, we will also need a reliable model for the steady state X-ray emission from the surrounding disk and corona, which will determine the relative contribution from the oscillating torus.

The power spectra in Figure 3, while reproducing the observed 3:2 frequency ratio of the QPO peaks, still do not agree quantitatively with the data from systems such as XTE J1550-564 and H1743-322 (Remillard et al. 2002; Homan et al. 2005). In those systems, the QPO frequencies are higher, the peaks are broader, and the ratio of the peak amplitudes is smaller. While a future paper will survey the model parameter space more comprehensively, we suggest here a few factors that we believe will help reconcile these differences. First, by including black hole spin, the torus radius will decrease, in 


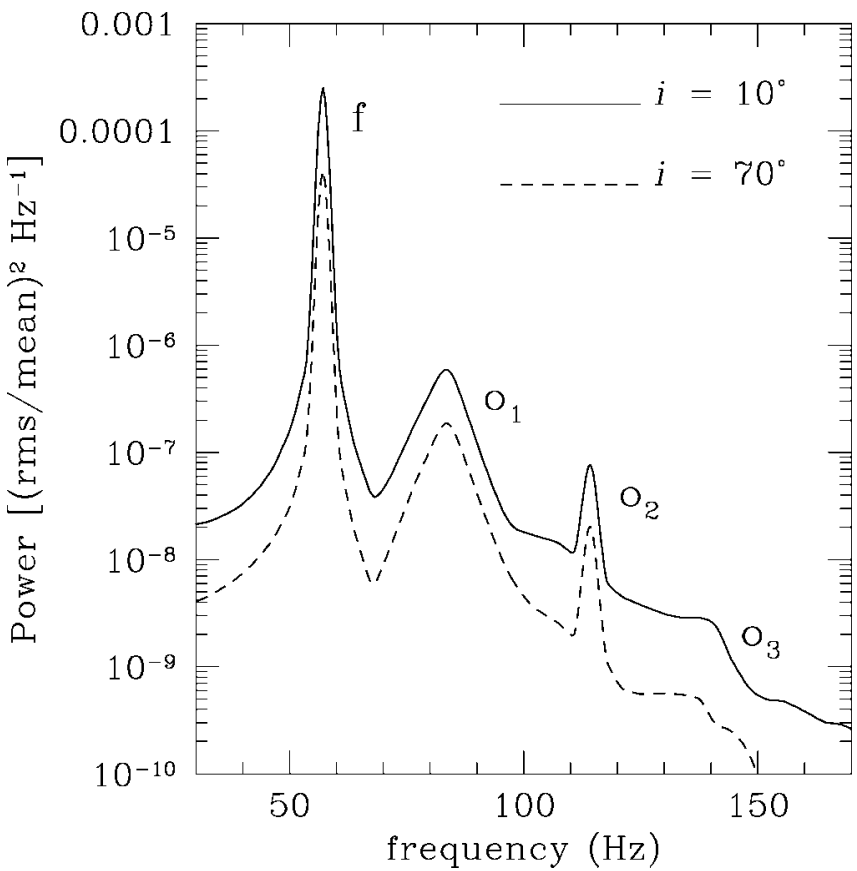

FIG. 3.-Power spectra of the light curves plotted in Fig. $2 a$, for a Schwarzschild black hole with mass $M=10 M_{\odot}$. The harmonic peaks are located at frequencies with integer ratios, with $o_{1} \simeq(3 / 2) f, o_{2} \simeq 2 f$, and $o_{3} \simeq$ $(5 / 2) f$.

turn increasing the oscillation frequencies for a given mass. In addition, by exciting different modes in the initial torus, the peaks of the power spectrum will have significantly different amplitudes (see Fig. 3 of Rezzolla et al. [2003] and Fig. 5 of Zanotti et al. [2005]). In particular, we believe that it should possible for the two peaks to have similar power, in closer agreement with the observations. Furthermore, in a real accreting system it is likely that any perturbation will last only a short time, as the turbulent conditions of the accretion disk would continually create and destroy coherent oscillating tori with random phases and amplitudes. We have found that these shorter lived perturbations could maintain more significant power in the higher harmonics than those shown in Figure 3. Lastly, the discontinuous phase shift between subsequent tori provides a natural explanation for the broadening of the QPO peaks. Indeed, a characteristic torus lifetime of only three to five periods is sufficient to explain the observed oscillator quality factors of $Q \approx 10-15$ (Schnittman 2005).

\section{DISCUSSION AND CONCLUSIONS}

We have demonstrated a positive correlation between the intrinsic normal-mode oscillations of a pressure-supported torus and the extrinsic X-ray light curves and power spectra as seen by a distant observer. In addition to this being the first raytracing calculation exploiting dynamically the results of relativistic hydrodynamics simulations, our investigation confirms the feasibility of the oscillating-torus model as an explanation for the integer ratios seen in high-frequency QPO peaks. The specific parameters of the torus model still require further investigation in order to best fit the QPO data, including a more comprehensive study of black hole mass, spin, and inclination angles.

For the line emission models considered, the variation in the light curve is caused largely by the gravitational redshift of photons coming from different radii as the torus moves in and out of the black hole's potential well. Unlike the relativistic hot-spot model, for the same emission mechanism the oscillating-torus model predicts higher amplitude variations in the light curve for smaller inclination angles, while at higher angles the special relativistic beaming and gravitational lensing counter the gravitational redshift, reducing the variations in flux. On the other hand, the thermal emission model predicts greater emission at small radii and, thus, larger amplitude variations for higher inclinations. These differences could be crucial to distinguish various QPO and emission models as more observations become available. An accurate model of the background disk and corona emission will also be essential for predicting the relative contribution from the oscillating torus to the total emission. This ratio will in turn determine the amplitude of the QPO peaks in the observed power spectrum.

Work is in progress to expand the results presented here by analyzing the dynamics of the torus in a Kerr spacetime, simulating a broader range of torus initial conditions, and including many short-lived oscillations with random phases. With these additions, we should be able to compare our simulated light curves and power spectra directly with other theoretical models and RXTE observations, matching the frequencies, widths, and in particular the amplitudes of the QPO peaks.

J. D. S. is grateful to E. Bertschinger for his insights and encouragement. Helpful discussions with O. Zanotti are also acknowledged. Support for this work was provided by NASA grant NAG 5-13306.

\section{REFERENCES}

Abramowicz, M. A., \& Kluźniak, W. 2001, A\&A, 374, L19

Beckwith, K., \& Done, C. 2004, MNRAS, 352, 353

Bursa, M., Abramowicz, M. A., Karas, V., \& Kluźniak, W. 2004, ApJ, 617, L45

Font, J. A., \& Daigne, F. 2002, MNRAS, 334, 383

Homan, J., Miller, J. M., Wijnands, van der Klis, M., Belloni, T., Steeghs, D., \& Lewin, W. H. G. 2005, ApJ, 623, 383

Lee, W. H., Abramowicz, M. A., \& Kluźniak, W. 2004, ApJ, 603, L93

Michel, F. C. 1972 , Ap\&SS, 15, 153

Miller, J. M., et al. 2001, ApJ, 563, 928

Montero, P. J., Rezzolla, L., \& Yoshida, S. 2004, MNRAS, 354, 1040

Nowak, M. A., Wagoner, R. V., Begelman, M. C., \& Lehr, D. E. 1997, ApJ, 477, L91

Okazaki, A. T., Kato, S., \& Fukue, J. 1987, PASJ, 39, 457

Remillard, R. A., Muno, M. P., McClintock, J. E., \& Orosz, J. A. 2002, ApJ, 580,1030
Rezzolla, L., Yoshida, S., Maccarone, T. J., \& Zanotti, O. 2003a, MNRAS, 344, L37

Rezzolla, L., Yoshida, S., \& Zanotti, O. 2003b, MNRAS, 344, 978

Rybicki, G. B., \& Lightman, A. P. 1979, Radiative Processes in Astrophysics (New York: Wiley)

Schnittman, J. D. 2005, ApJ, 621, 940

Schnittman, J. D., \& Bertschinger, E. 2004, ApJ, 606, 1098

Schnittman, J. D., Homan, J., \& Miller, J. M. 2006, ApJ, in press (astro-ph/ 0512595)

Stella, L., \& Vietri, M. 1999, Phys. Rev. Lett., 82, 17

Strohmayer, T. E. 2001, ApJ, 552, L49

Zanotti, O., Font, J. A., Rezzolla, L., \& Montero, P. J. 2005, MNRAS, 356, 1371

Zanotti, O., Rezzolla, L., \& Font, J. A. 2003, MNRAS, 341, 832 